\section{EMBRYAIDDLE Aeronautical University}

SCHOLARLY COMMONS
International Journal of Aviation, Aeronautics, and Aerospace

\title{
Active fault-tolerance of the unmanned aerial vehicle automatic control systems
}

\author{
Vuong Anh Trung \\ Air Defense - Air Force Academy, Son Tay, Ha Noi, Viet Nam, vuonganhtrung@gmail.com \\ Nguyen Van Thinh \\ Air Defense - Air Force Academy, Son Tay, Ha Noi, Viet Nam, Thinhkthk@gmail.com \\ Nguyen Duc Thanh \\ Academy of Military Science and Technology, Ha Noi, Viet Nam, thanhnd37565533@gmail.com \\ Nguyen Quang Vinh \\ Academy of Military Science and Technology, Ha Noi, Viet Nam, vinhquang2808@yahoo.com \\ Tran Thuan Hoang \\ Faculty of Electrical-Electronic Engineering, Duy Tan University, Danang, 550000, Vietnam; Institute of \\ Research and Development, Duy Tan University, Danang, 550000, tranthuanhoang@duytan.edu.vn
}

Follow this and additional works at: https://commons.erau.edu/ijaaa

Part of the Aerodynamics and Fluid Mechanics Commons, Navigation, Guidance, Control and Dynamics Commons, and the Propulsion and Power Commons

\section{Scholarly Commons Citation}

Trung, V., Thinh, N., Thanh, N., Vinh, N., \& Hoang, T. (2020). Active fault-tolerance of the unmanned aerial vehicle automatic control systems. International Journal of Aviation, Aeronautics, and Aerospace, 7(4). https://doi.org/10.15394/ijaaa.2020.1538

This Article is brought to you for free and open access by the Journals at Scholarly Commons. It has been accepted for inclusion in International Journal of Aviation, Aeronautics, and Aerospace by an authorized administrator of Scholarly Commons. For more information, please contact commons@erau.edu. 
Modern UAV automatic control systems are complicated, integral, intelligent, and multifunctional. Those systems are composed of different devices and elements, determining a higher level of synthesis problems for a UAV automatic control system. The essential scale, heterogeneity, and uniqueness of these systems are the reason why they are so vulnerable to such destabilizing moments as breakdowns, defects, and errors, which are generally called faults. Caused by faults, abnormal situations corroborate the imperfection of designed UAV ACS. The main reason for the deficiency is a system's low "intelligent level" applied during fault parrying. Such level increasing can be achieved by using deeper fault-diagnosis, more flexible failure toleration or, in a word, by implementing active fault-tolerance. Using active fault-tolerant control instead of majority logic voting opens new possibilities to the essential decrease of weight, size, cost, and energetic system specifications and to increase guaranteed operability period and, therefore, increase the effectiveness of project UAV ACS at a whole. Research on the system's active fault-tolerance have had some history, and these are presented in (Alwi et al., 2014; Iserrmann, 2004; Li, 2009; Zhang \& Jiang, 2008). However, the proposed models, methods and tools to ensure the active fault-tolerance are predominantly fragmented, and they do not reflect the dynamics of the control processes, do not consider the possibilities of diagnosing the operational state of the functional elements, as well as fault parrying through the effective use of existing and the introduction of additional redundancies. Consequently, the development of diagnosing models and methods, as well as failure parrying to provide an active faulttolerance of the UAV attitude determination and control in real-time, is a significant scientific and applied problem.

\section{Active Fault-Tolerance System Structure}

The first part of the structure of the three-layer hierarchy of active faulttolerance. Second is deep fault-diagnosis. And third is flexible failure toleration. Using the first principle leads to block, system, and over system levels of active fault-tolerance providing (Schneider, 1990). From the whole set of critical for system fault types $D=\left\{d_{i}\right\}_{1}^{q}$, fault types $d_{i} \in D_{1} \subset D$ that can be tolerated on the functional blocks (sensors, actuators, and controller) are chosen. The subset $D_{1}$ is formed in terms of available block redundancy. On the system level subset $D_{2} \subset D$ of fault types tolerated with the help of system redundancy. For the third over system set $D_{3} \subset D$, which contains level fault types tolerated with the help of such over system redundancy as changing functioning mode of UAV ACS is formed. 


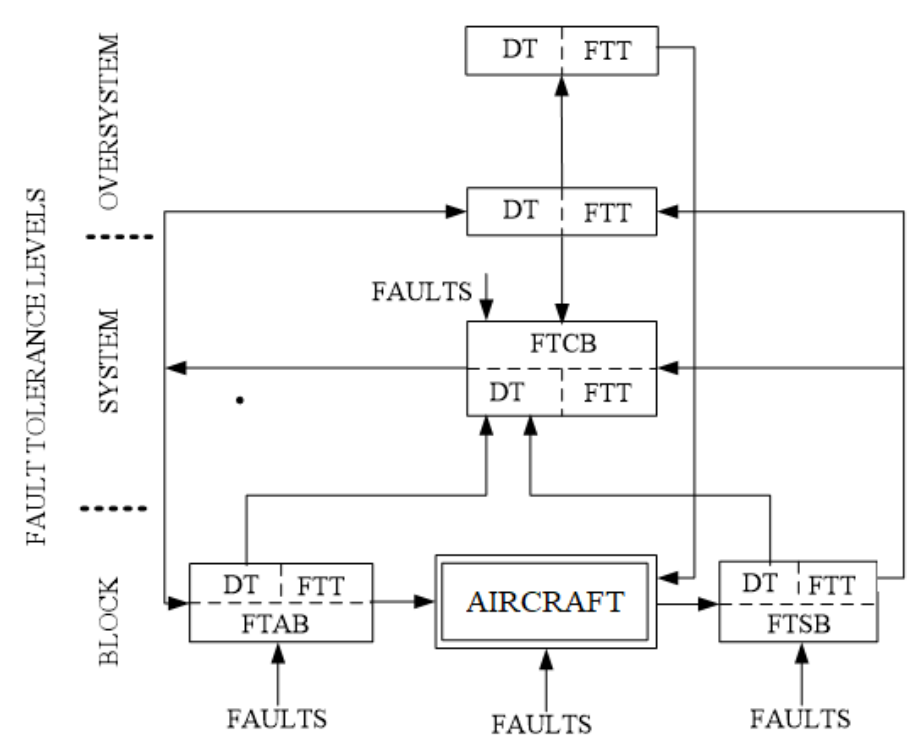

Figure 1. Block diagram of active fault-tolerant control in abnormal mode.

Deep fault-diagnosis consists in search of a concrete tolerated fault type $d_{i}, i=\overline{1, q}$ based on available measurements. Deep fault-diagnosis has proceeded on each level of fault-tolerance providing.

The principle of flexible failure toleration resides, informing for each fault type possible alternatives of going out from abnormal situations with the help of redundancy. Failure toleration procedures are formed in terms of object models functioning and by taking into account redundant resources (Benosman, 2010)

According to considered principles, new structure of UAV systems is needed. On the block level system is composed of UAV, fault-tolerant sensors block (FTSB), fault-tolerant actuators block (FTAB), and fault-tolerant controllers block (FTCB) that in turn includes diagnosis tools (DT) and failure toleration tools (FTT) used in abnormal modes. On the system-level, diagnosis and tolerating procedures are executing with the help of system redundant tools. On the third over, the system-level quality of lower levels functioning is diagnosed, and system operability is recovered by using available resources (Saved et al., 2016).

\section{Deep Fault-Diagnosis}

The development of deep fault-diagnosis procedures is based on two types of diagnostic models. First of them is a functional diagnostic model (FDM), which connects deviation of direct fault indications with symptoms, and second is a logical diagnostic model (LDM), which reflect qualitative linkage between symptoms. This way, the abnormal functioning mode of the linearized 
diagnostic object for "small" faults can be presented as followed FDM (Ducard, 2009; Marks et al., 2012; Vinh, 2017).

$$
\begin{aligned}
& \Delta x(k+1)=A \Delta x(k)+\left[A_{i} \hat{x}(k)+B_{i} u(k)\right] \Delta \lambda_{i}+\eta(k) ; \\
& \Delta x\left(k_{0}\right)=x_{0} ; \\
& \Delta y(k)=C \Delta x(k)+\left[C_{i} x(k)+D_{i} u(k)+F_{i}\right] \Delta \lambda_{i}+\mu(k),
\end{aligned}
$$

I called MD-model. Here $A_{i}, B_{i}, C_{i}, D_{i}$ and $F_{i}$ are the sensitivity functions of matrixes $A(\lambda), B(\lambda), C(\lambda), D(\lambda)$ and $F(\lambda)$ on diagnostic parameters $\lambda_{i} ; \eta(k)$ and $\mu(k)$ are errors and noise vectors; $\Delta x(k)$ are deviations of the state vector, and $\Delta y(k)$ are deviations of diagnosed object measurements vector. $\Delta \lambda_{i}=\lambda_{i}-\lambda_{\text {in }}$ Is a deviation of direct diagnostic indication from normal value $\lambda_{i n}$ ? From equation system (1), following that linear finite-difference equations describe MD-model with variable coefficients and the law of coefficients, varying is determined by the behaviour of reference model state vector $\mathrm{x}(\mathrm{k})$ and control action vector $u(k)$.

In the general case, the relationship between symptoms $\Delta y(k)$ and direct diagnostic indication can be presented with the help of followed equations (Beckman \& Aldrich, 2007).

$$
\Delta y(k)=\varphi_{i}(k) \Delta \lambda_{i} ; i=\overline{1, \mu},
$$

Where $\varphi_{i}(k)$ does sensitivity function correspond to the ith direct diagnostic indication? This function is a time-discrete function, and the transition to the qualitative indication of its numerical characteristics $\eta_{i j}$ are introduced. Thus, for the transition from absolute indications scale to designation scale, double-valued predicate equations are used:

$$
z_{j}=S_{2}\left(\eta_{i j}-\delta\right)=\left\{\begin{array}{l}
1, \text { if } \eta_{i j} \geq \delta \\
0, \text { if } \eta_{i j}<\delta_{j}, j=\overline{1, m},
\end{array}\right.
$$

S2(.) is a sign of a double-valued predicate, $\delta_{i}$ it is a threshold value dependent on presentation and processing data accuracy. By sequential use of equation (3), the Boolean matrix with the dimensions $\mu \times m$ is obtained. The number of matrix rows corresponds to the number of direct diagnostic indications 
$\Delta \lambda \mathrm{i}$, and the number of matrix columns corresponds to the measurement of symptoms vector $\Delta \mathrm{y}(\mathrm{k})$. This matrix presents by itself a kind of DLM called table logical model (TL-model).

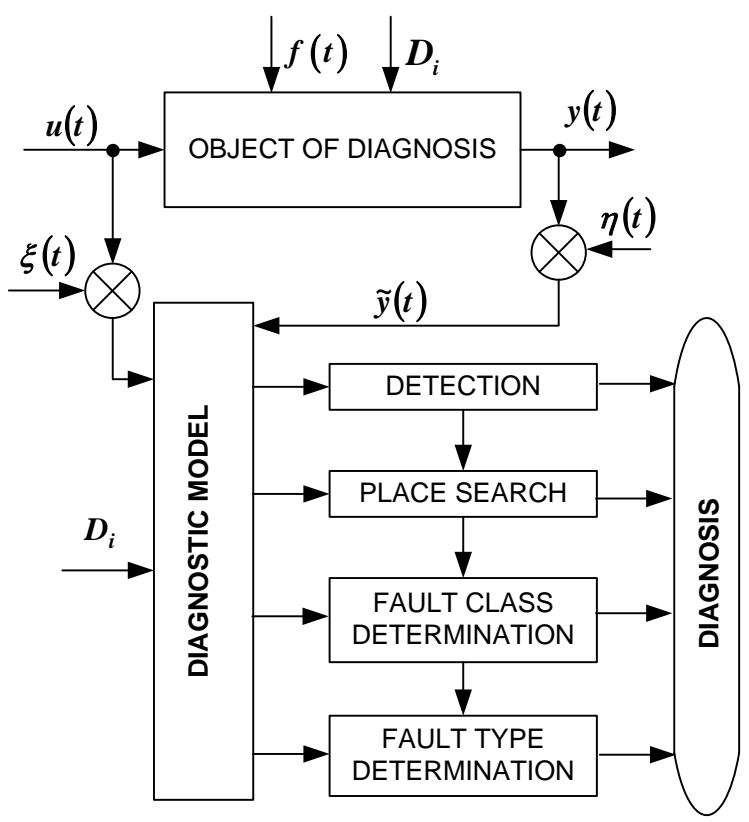

Figure 2. Deep signal-parametric diagnosis schema.

Mathematical FDM and LDM are developed for linear nonlinear systems classes and systems with essential nonlinearities for "small" and "big" faults. Diagnostic models allowed to create the new signal-parametric approach that permitted to solve analytically such main tasks of deep fault-diagnosis as fault detection, fault place search, fault class determination, and fault type determination (see Figure 2).

The signal-parametric approach is based on the assumption that the appearance of any fault kind $d_{i} \in D$ is an indefinite event in the view of the moment of fault appearance, place fault from, class of fault and concrete kind of responsibility. Such event uncertainty taking down is connected with calculating characteristics based on measurable system symptoms that correspond to each task. As a base structure for sequential delay taking down in abnormal situations, a dichotomic tree search was chosen. Dichotomic trees are built with LDM using and present by itself base on formation computer production knowledge base of the in-depth diagnosis process. 


\section{Flexible Failure Toleration}

The main reasons for abnormal modes appearance in UAV systems are sensors, actuators, and faults of UAV. Appearance faults in the sensor lead to distortions or losses of measuring data. That is why fault parrying, in this case, is concluded in measuring recovery with the help of existing hardware and redundant software resources.

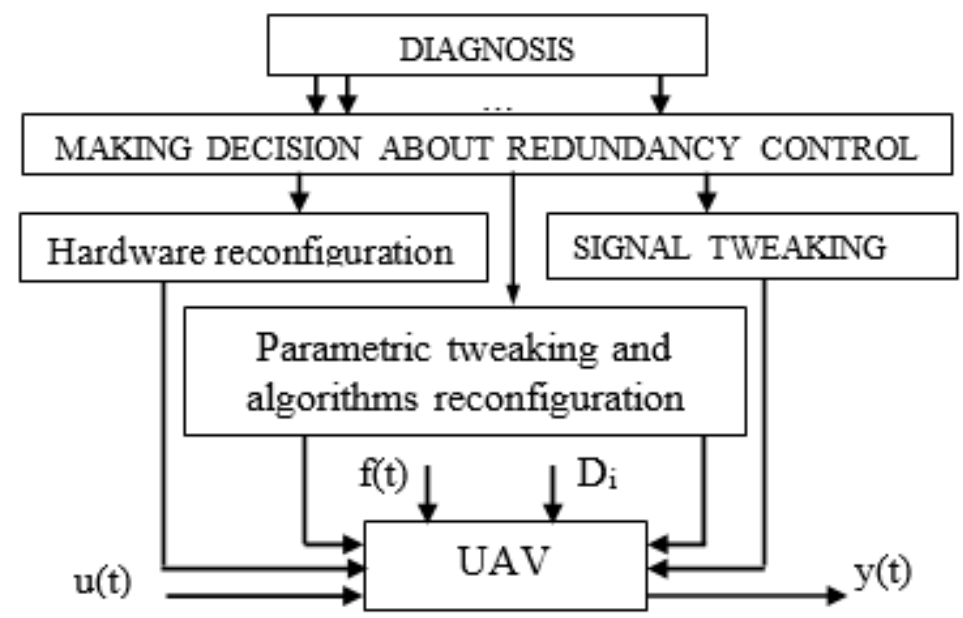

Figure 3. Parrying tools of flexible failure UAV ACS at the system level.

On abnormal work of actuators, operability recovery of the whole system is made by particular use of redundant sensors, actuators and by parametric and signal tweaking. ACS faults can be tolerated by algorithmic tools. Defects that appeared in UAV ACS can be presented by two main classes: compensable and not compensable. For accepting compensable faults, methods and means of parametric and signal tweaking are created. Not compensable are tolerated by control algorithm reconfiguration and redundant hardware.

For synthesis algorithms of tolerating faults, a discrete analogue of second A.M. Lyapunov's method was used. The reason why this method was chosen is the possibility to do structural and parametric UAV ACS failure to tolerate algorithms synthesis and to provide a classical condition of operability steadiness.

\section{Experimental Research}

Debugging functional algorithms of diagnosing applicable state and recovery of the object autonomic control (OAC) of the UAV performed on a specialized hardware-software complex (HSC), presented in Figure 4. 
The functional structure of HSC allows us to enter into the system failure modes from the considered set and to investigate the behaviour of the system in the nominal and emergency ways.

HSC consists of the following elements: a UAV model with all on-board equipment, a strap-down inertial navigation system (SINS) with a minimally redundant sensor unit, established by pyramid scheme, an automatic control device and the PC with specialized software (Anh et al., 2019; Ding, 2008; Milhim et al., 2011; Pupkov \&Vinh, 2006).

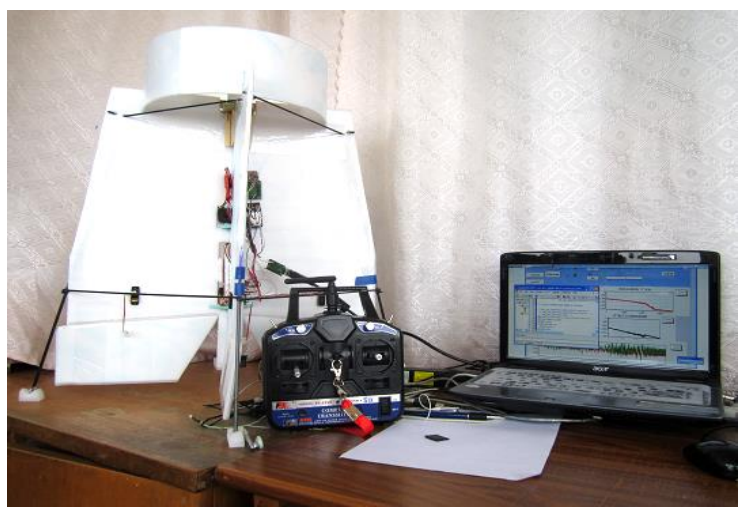

Figure 4. Exterior view of HSC for research models and methods of maintaining the active fault-tolerance UAV ACS.

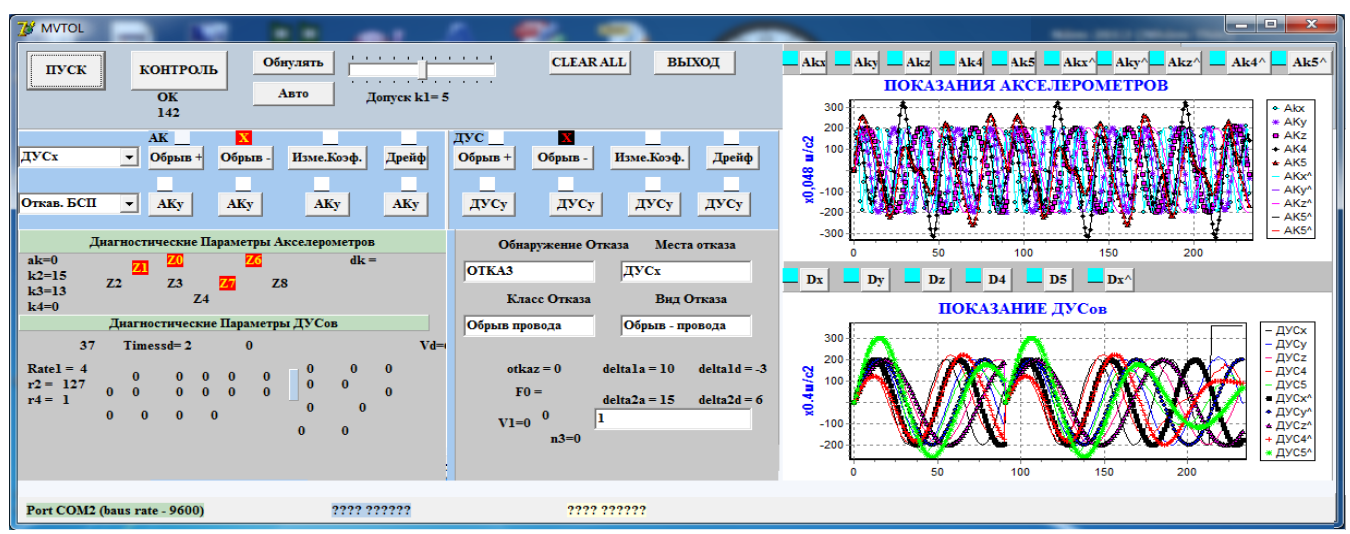

Figure 5. The interface of the program results and operational mode for the ACS.

The complex software includes low-level software that provides execution in the control microcontroller module and the peripheral 
microcontroller module developed procedures control of and provide the active fault tolerance. On the upper level, there is software for performing the following functions: information exchange with an automatic control device, setting modes of the system, visualization, and storage of the experimental results.

To simulate the effect of the considered set of failure modes on the system using developed HSC uses software simulators. They are used because they have essential advantages such as the possibility of rapid modification, imitation of a wide variety operating conditions, ease of the results interpretation, small power consumption, good adaptability at this distortion of measured and control signals of the system performed so that the response to it was an analogous reaction to input types of failure.

Figure 6 shows the results of UAV ACS simulation without active fault tolerance (bold line) and with an activated diagnosis and recovery system (thin line) at the mode of the angular velocity of inertial navigation systems on an axis. Introduces the following notation for times: to - introduction failure to the system; tod - failure detection system; ts - the beginning of recovery by the signal adjustment; $\mathrm{tf}$ - time of complete restore.

Comprehensive research of the control system on a given set of failures shows that all types of failure were observed by diagnostics the subsystem, determining the location of the class and kind of failure and obtained estimations of the direct attributes of failures. The average recovery time was trec $=2,4 \mathrm{~s}$.

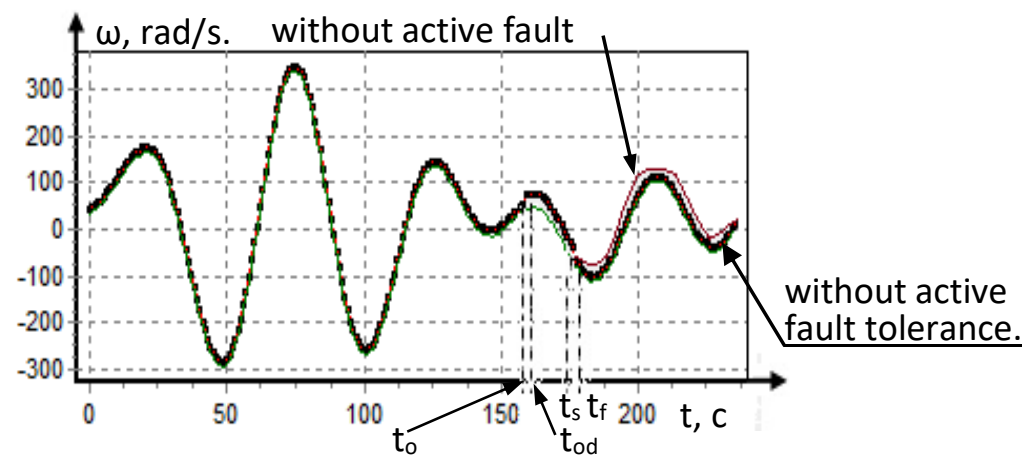

Figure 6. The results of the UAV ACS with the drift of angular velocity sensor.

Experimental investigation of diagnosis and recovery from an angular sensor drift. Similarly, research has been done in the modes of attitude control and stabilization object functioning on the whole parrying failure modes set. All types of failure were observed by the diagnostics subsystem, found their place, set the class, and defined failure mode. 
Investigations on the fault tolerant actuators block mock-up that consists of other elements that were conducted similarly. On the scaled-down modelling mock-up, abnormal situations called by UAV construction faults were examined, and fault diagnosis and UAV failure toleration procedures were finetuned. The results of experimental research show the constructiveness of the proposed conception, the effectiveness of used methods and tools for providing active fault-tolerance of UAV ACS during mock-up experiments.

\section{Conclusions}

Thus, in this paper identified the main trends in modern aviation technology that is in reducing the weight and size, power and cost characteristics, the function of aviation vehicles, as well as increasing the time of their active life. There was formed the structure of ACS for ensuring its dynamic fault tolerance feature, defined a set of standard and parameterized types of failures of the system functional elements, characterized by changes in the properties of the conversion elements. There was received diagnostic functional models for solving problems of detection, search the place and establish a class of failure, which provide unique analytical relation between direct and indirect diagnostic features for each diagnosing problem, take into account the dynamic features of the object, as well as systemic linkages between its elements. There were developed diagnostic logic models and methods of a systematic approach to fault tolerance in the direction of the effective use of the signal, parametric and structural redundancies and selection of parrying tools when there are multiple connections between the means of parrying and failure modes. Experimental researches of the sample model of ACS the properties of active fault-tolerance in emergency modes of operation which have shown operability of the developed models and methods of deep diagnosing and failures flexible parrying and fundamental possibility maintain operability of the object in the event of failure modes in it from the finished set.

\section{Acknowledgement}

The authors would like to thank the School of engineering technology, Duy Tan University, Danang, Vietnam. 


\section{References}

Alwi, H., Humayun, M., \& Edwards, C. (2014). An integral sliding mode faulttolerant control scheme for an octocopter using control allocation. 13th International Workshop on Variable Structure Systems (VSS), 1-6. doi:10.1109/ACC.2013.6580859

Anh, P. T. P., Vu, N., Lai, P. T., \& Vinh, N.Q. (2019). Sliding mode based lateral control of unmanned aerial vehicles. Procedia Computer Science, 150, 78-87.

Beckman, N., \& Aldrich, J. (2007) A programming model for failure-prone, collaborative robots. In Proceedings from Workshop on Software Development and Integration in Robotics. Retrieved from https://www.semanticscholar.org/paper/A-Programming-model-forfailure-prone-\%2C-robots-Beckman-Aldrich/ 49c995ed575630e86209b135c568c79a99eb2d4d

Benosman, M. (2010). A survey of some recent results on nonlinear faulttolerant control. Mathematical Problems in Engineering, 1-25.

Ding, S. X. (2008). Model-based fault diagnosis techniques: Design schemes, algorithms, and tools. Springer, Heidelberg.

Ducard, G. (2009). Fault-tolerant flight control and guidance systems: Practical methods for small unmanned aerial vehicles. London: Springer.

Isermann, R. (2004). Model-based fault detection and diagnosis - status and applications. Annual Reviews in Control, 71-85.

Li, X. (2009). Fault detection filter design for linear systems [Ph.D. Dissertation, Louisiana State University]. Retrieved from https://digitalcommons.lsu.edu/cgi/viewcontent.cgi? article $=2836 \&$ contex $t=$ gradschool_dissertations

Marks, A., Whidborne, J. F., \& Yamamoto, I. (2012). Control allocation for fault-tolerant control of a VTOL octocopter. UKACC International Conference on Control, 357-362. doi:10.1109/CONTROL.2012.6334656

Milhim, A. B., Zhang, Y. M., \& Rabbath, C. A. (2011) Gain scheduling based PID controller for fault tolerant control of a quad-rotor UAV. AIAA Infotech@Aerospace, April. doi:10.2514/6.2010-3530

Pupkov, K. A., \& Vinh, N. Q. (2006) Analysis of the self-guidance precision with maneuvering target and control nonlinearities. Journal Science Intensive Technologies, 10, 8-19.

Saved, M., Lussier, B., Fantoni, I., Shraim, H. \& Francis, C. (2016) Passive fault-tolerant control of an octocopter using the super-twisting algorithm: theory and experiments. 3rd Conference on Control and FaultTolerant Systems (SysTol). https://systol16.cs2ac.upc.edu/

Schneider. F. B. (1990). Implementing fault-tolerant services using the state machine approach: A tutorial. ACM Computing Surveys, 22(4), 299-319. 
Vinh, N. Q. (2017). Combination of an inertial measurement unit and a camera for defining the position of an airplane. Science Journal of Applied Mathematics and Statistics, 5, 78-85.

Zhang, Y. M., \& Jiang, J. (2008). Bibliographical review on reconfigurable fault-tolerant control systems. Annual Reviews in Control, 32(2), 229252. 